\title{
Periodic Pulling Oscillation in Air Glow Discharge at Low Pressure
}

\author{
Ahmed Y. Owaid \\ \{ahmedowaid69@gmail.com, ahmedowaidphysics@ntu.edu.iq\} \\ Technical Institute of Mosul, Northern Technical University, Mosul, Iraq
}

\begin{abstract}
The incomplete response of a nonlinear oscillatory system to a periodically driving force called periodic pulling oscillation. In the present study, this phenomenon is observed in the discharge current of airglow discharge that generated at low pressure (55$85 \mathrm{mT}$ Trr) by a periodically driving force (a sinusoidal voltage) of the period $(3.33 \mathrm{mSec}$ ). The glow discharge is created between two plan electrodes of Aluminum, sited parallel inside a Pyrex cylindrical tube at the distance $(9.5 \mathrm{~cm})$. It has been observed that the periodic pulling waveform and amplitude changed with the discharge pressure and voltage. A maximum periodic pulling is obtained at a moderate discharges voltage and pressure, namely at normal glow discharge voltage and pressure that coincides with the pressure of the Paschen curve minimum. Furthermore, it is observed that the periodic pulling oscillation is weak and disappear at a higher discharge voltage and /or discharge pressure within the range.
\end{abstract}

Keywords: Periodic pulling oscillation, periodic pulling, airglow discharge, and lowpressure discharge.

\section{Introduction}

Gases discharge plasma has wide applications in the medicinal and engineering devices technology. One of the important features of those devices is discharge stability. Oscillations represent a well-known type of discharge instabilities, which have a variety of reasons, It is often a self-enhanced, initially random fluctuations in electron and ion loss rates to the chamber walls, a sheath resonance instabilities, and signals that picked up from a plasma source. The oscillations could also be related to a mixture of the instability processes occurring within the discharge itself and the external discharge circuit. Instabilities are observed in all kinds of plasma, ranging from the low-density plasma of Townsend discharges up to high-density Tokomaks plasmas. Many studies have been shown that there are continuous or damped oscillations in a discharge current at the stable, pulsed, or periodic discharge voltage [1-5]. Earlier, an outsized effort has made to know the reasons of discharge current oscillations [8]. In the Townsend discharge, it is observed that the space charge causes a negative resistance reinforces an oscillation within the discharge current [9]. To explain the discharge instabilities, phenomenological models often considered the foremost effective circuit elements [11,12], while physical models take the present and distribution of charged particles within the discharge chamber $[9,13]$. The oscillations in the discharge current could be of the relaxation type when the discharge turning off and on repeatedly through the transitions between different modes of operation [15]. Periodic pulling is another type of a nonlinear oscillation that occurs in the 
discharge current due to the incomplete response of the discharge to periodic drive action. During that, the system oscillation stops the synchronization at regular intervals, and the oscillation frequency pulled toward the driving frequency. The periodic pulling produces a kind of AM and FM in the system oscillatory response. These combined modulations produced an envelope of asymmetric sides. The periodic pulling phenomenon is observed in many physical systems [6-11]. In plasma, the periodic pulling has been investigated in a bounded plasma, beam-plasma interactions, a He-Ne laser within a weak magnetic field, and in avalanche-diode oscillators [12-15]. In the present study, a sufficient periodically driving force (sinusoidal voltage) is used to verify the periodic pulling in the current waveform of airglow discharge at low pressures. It is observed that a maximum periodic pulling is obtained at a moderate driving voltage and a low pressure which coincides with that of the Paschen curve minimum. It is also shown that the periodic pulling oscillation weakens with increasing the discharge voltage and eventually disappear with increasing the discharge pressure.

\section{Experimental Setup}

The experimental arrangement of this study is shown in Figure 1. The glow discharge of air is created between two flat disc Aluminum electrodes separated by $(9.5 \mathrm{~cm})$ inside a Pyrex cylindrical tube of diameter $7.5 \mathrm{~cm}$. The parts of the discharge chamber are cleaned and dried carefully before they used. A rotary vacuum pump with a thermocouple vacuum gauge is used to control the pressure of air in the range (55-85 mTorr).

The glow discharge of air is generated and derived periodically by a different value (350$600 \mathrm{~V})$ of a sinusoidal voltage of the time $(3.33 \mathrm{mSec})$. The driving voltage is supplied from a high voltage power supply capable to supply up to $700 \mathrm{~V}$ of $250 \mathrm{~mA}$. A ballast $330 \Omega$ resistor of $1.5 \mathrm{~W}$ is used in series in the discharge circuit to limit the discharge current. A digital storage oscilloscope of high impedance (Hantek DSO5072P) was used to capture the discharge current waveform images. The images have been treated by Matlab image processing tools to get the periodic pulling data, which are also used in the Matlab curve fitting tools to obtain the periodic pulling waveform. Keep in mind that all voltages that will state in the results and figures represent the root mean square values.

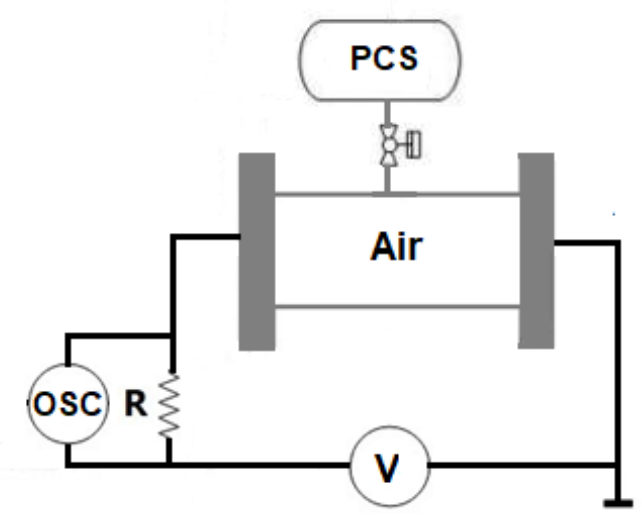

Fig. 1. Schematic of airglow discharge system, PCS (pressure control system), OSC (oscilloscope), V (discharge and driving voltage), and $\mathrm{R}$ (ballast resistor). 


\section{Results and Discussion}

Figure 2 shows an image of a periodic pulling that observed in the discharge current waveform of airglow discharge. The periodic pulling waveform (the modulation signal or the envelope) was fitted by Matlab to the Fourier series that given by the following equation [17].

$$
y=a_{0}+\sum_{k=1}^{n} a_{k} \cos (k \omega t)+b_{k} \sin (k \omega t)
$$

Where $y, \omega, \mathrm{n}$ and $a_{0}$ are the parameters of the periodic pulling waveform, the instantaneous amplitude, the fundamental frequency, the number of harmonics and the DC component respectively.

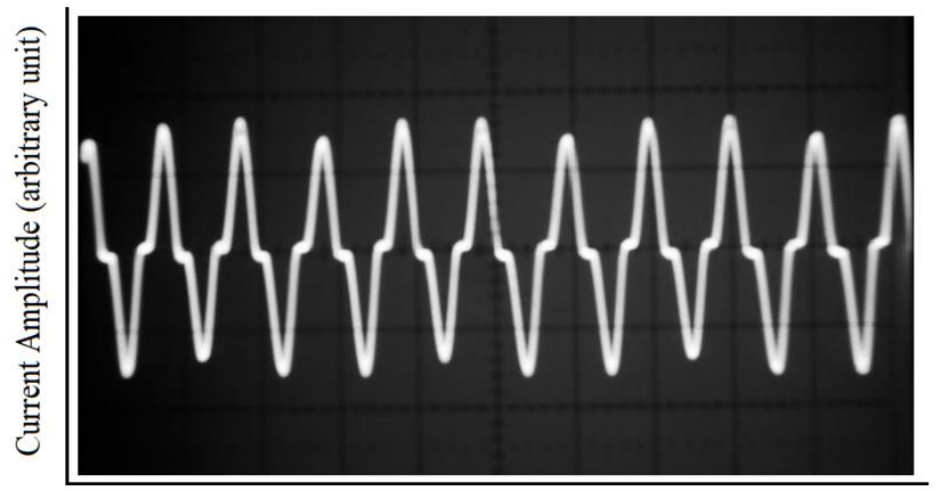

Time (arbitrary unit)

Fig. 2. An image of a periodic pulling in the discharge current waveform, captured at pressure (65 mTorr) and driving discharge voltage $(378 \mathrm{~V})$ of the period of time $(3.33 \mathrm{mSec})$

At a discharge pressure in the range (55-85 mTorr) and/or a discharge voltage in the range (350$600 \mathrm{~V}$ ), the periodic pulling waveform (modulation signal) has a specific waveform, time and oscillation amplitude. Figure 3 illustrates the periodic pulling waveform with it is equivalent Harmonics observed in the discharge current at different conditions (discharge voltage and pressure). The periodic driving voltage impresses the discharge to oscillate between two modes of operation, glow discharge mode (generation of charged particles) and dark discharge mode (loss of charged particles). A number of charged particles is leftover in the discharge chamber during the transition from glow to dark mode. The density of the remaining charged particles is not constant and it changed in some way at each half cycle of the driving voltage (oscillate between different values). The periodic pulling oscillation was formed in the discharge current by the oscillation of charged particle density that leftover in the discharge chamber during the periodic transitions. The net rate of generation and loss of charged particles in a mode of discharge is given by equations (2) and (3), for electrons and ions respectively [18, 21].

$$
\begin{gathered}
\frac{\partial n_{e}}{\partial t}=I_{R} N n_{e} \exp \left(-\frac{E_{i}}{K_{B} T_{e}}\right)-\mu_{e} \nabla\left(n_{e} \nabla V\right)+D_{e} \nabla^{2} n_{e} \\
\frac{\partial n_{i}}{\partial t}=I_{R} N n_{e} \exp \left(-\frac{E_{i}}{K_{B} T_{e}}\right)+\mu_{i} \nabla\left(n_{i} \nabla V\right)+D_{i} \nabla^{2} n_{i}
\end{gathered}
$$


Where $n_{e}, \mu_{e}, D_{e}, n_{i}, \mu_{i}, D_{i}$ are the electron and ion parameters, density, mobility, and diffusivity respectively and $V, N, I_{R}, E_{i}, K_{B}$ and $T_{e}$ are discharge voltage, gas density, ionization rate, ionization energy, Boltzmann constant, and electron temperature respectively. Equations (2) and (3) have no analytical solution. It is clear that the predominant factors in the equations are gas density (gas pressure) and discharge voltage.

It is observed here experimentally that the periodic pulling oscillation can be seen clearly at low and moderate pressure and/or discharge voltage. Figure 4 displays the amplitude of the periodic pulling waveform at the values of discharge voltage and air pressure shown in table 1 . It is fairly from this figure that a maximum periodic pulling amplitude occurs at the pressure or $p d$ value that coincides with that of the Paschen curve minimum $(0.065$ Torr $\times 9.25 \mathrm{~cm}=$ 0.6 Torr. $\mathrm{cm}$ ), where $p$ is the discharge pressure and $d$ is the inter-electrodes distance [18]. It is also displayed in the same figure that the high oscillation amplitude occurs at a normal glow discharge voltage. The figure moreover, shows that the oscillation amplitude decreased sharply at low and high discharge voltage and finally disappear with increasing the discharge pressure at a given discharge voltage. It appears that the high pressure acts as a damping factor in the periodic pulling oscillations by increases the collision frequency between all particles. The high voltage could also be damping that oscillations by speed up the charged particles and so on the collision frequency of them will increase.
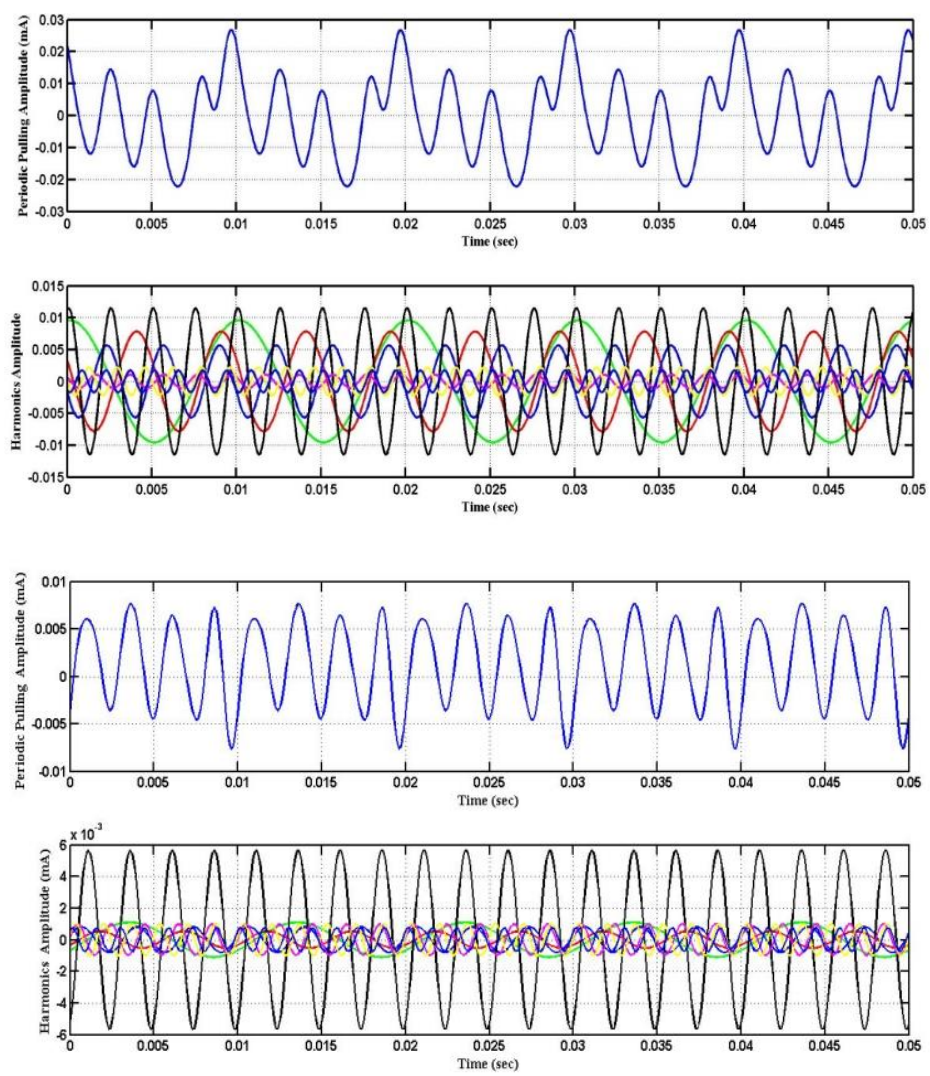

Fig. 3. Periodic pulling waveform with it harmonics obtained at top/ pressure ( $65 \mathrm{mTorr})$ and discharge voltage $(378 \mathrm{~V})$, bottom/ pressure (75 mTorr) and discharge voltage (430) 
Table 1. discharge pressures with discharge voltages sequence

\begin{tabular}{|c|c|c|c|c|c|}
\hline \multirow{2}{*}{$\begin{array}{c}\text { Discharge } \\
\text { Pressure (mTorr) }\end{array}$} & \multicolumn{5}{|c|}{ Discharge Voltage (Volt) } \\
\cline { 2 - 6 } & \multicolumn{5}{|c|}{ Discharge Voltage Sequence } \\
\cline { 2 - 6 } & 1 & 2 & 3 & 4 & 5 \\
\hline 55 & 460 & 489 & 502 & 523 & 547 \\
\hline 65 & 369 & 378 & 405 & 431 & 456 \\
\hline 75 & 430 & 503 & 517 & 534 & 546 \\
\hline 85 & 500 & 516 & 537 & 542 & 553 \\
\hline
\end{tabular}

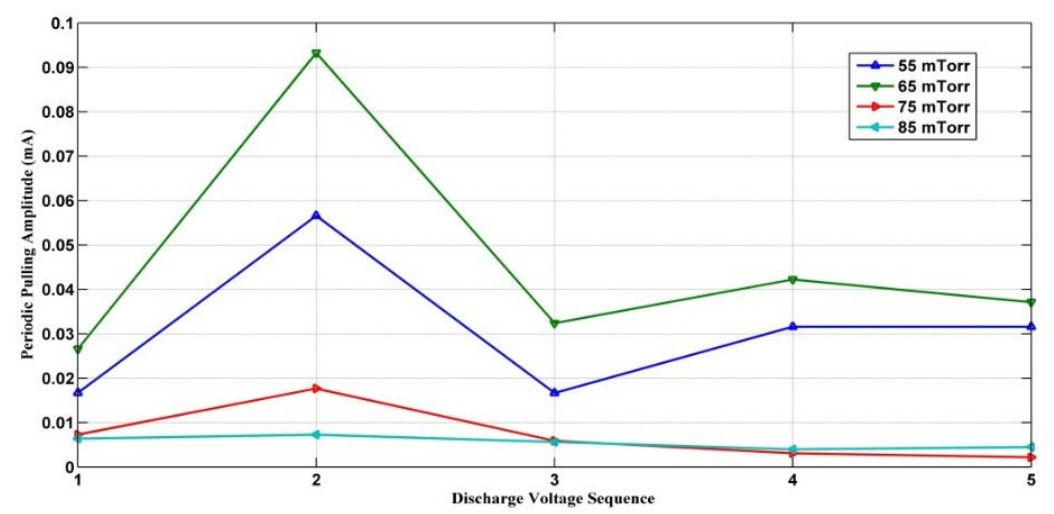

Fig. 4. Periodic pulling waveform amplitude at different discharge voltage and pressure

Figure 5 shows the frequency of the periodic pulling waveform at different discharge voltage and pressure. It is clear that the oscillation frequency of a periodic pulling decrease with increasing the discharge voltage and/or discharge pressure. This result is also produced by the effect of discharge pressure and voltage on the collision frequency of charged particles that generate the periodic pulling oscillation. Moreover, the figure shows that the frequency of a maximum periodic pulling is near (1/3) of that of the driving force (discharge voltage).

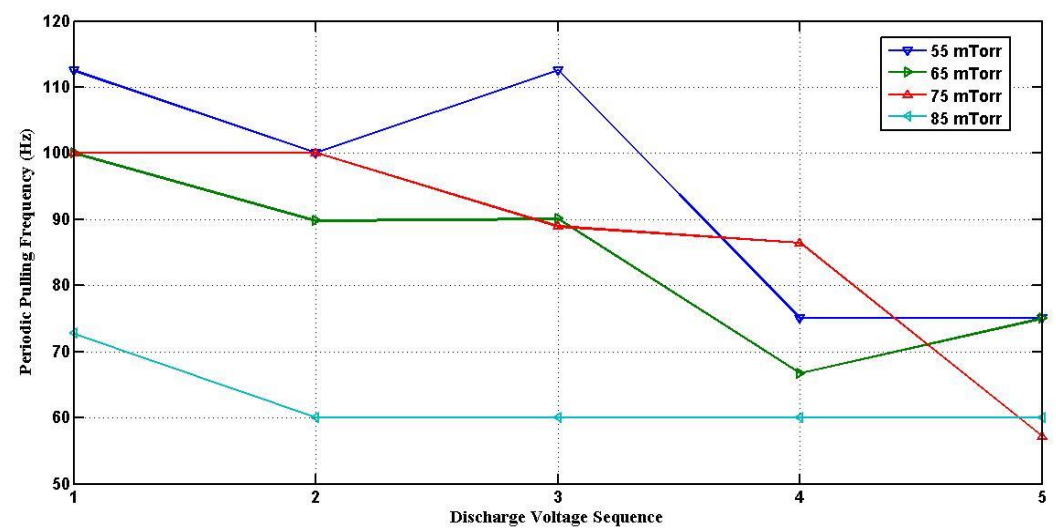

Fig. 5. Periodic pulling waveform frequency at different pressure and discharge voltage 


\section{Conclusions}

In the ranges (55-85 mTorr) and (350-600 V) of discharge pressure and voltage respectively, the periodic pulling has been observed in the airglow discharge current waveform. Measurements illustrate that the periodic pulling waveform has a specific waveform, time period, and oscillation amplitude that changed with discharge voltage and pressure. It is shown that a maximum periodic pulling occurs at a normal glow discharge voltage and a pressure that coincides with that of the Paschen curve minimum. The last two factors provide optimum conditions for periodic pulling oscillations (a high density of a leftover charged particle and a low collision frequency) in comparison with other values. Furthermore, it is displayed that the periodic pulling decreased with increasing the discharge voltage and finally disappear with increasing the discharge pressure. It is also shown that the periodic pulling oscillation frequency decreases with increasing the discharge voltage and/or discharge pressure and the frequency of a maximum periodic pulling is near (1/3) of the driving force (discharge voltage) frequency.

\section{References}

[1] Koepke, M. E. and Hartley, D. M.: Experimental verification of periodic pulling in a nonlinear electronic oscillator. Phys. Rev. A 44. 6877 (1991)

[2] Cunningham, W. J.: Introduction to Nonlinear Analysis. McGraw-Hill, New York. (1958)

[3] Guckenheimer, J. and Holmes, P.: Nonlinear Oscillations, Dynamical Systems, and Bifurcations of Vector Fields. Springer-Verlag, New York. (1983)

[4] Field, R. J. and Burger, M.: Oscillations and Traveling waves in Chemical Systems. Wiley, New York(1985)

[5] Pavlidis, T.: Biological Oscillators: Their Mathematical Analysis. Academic, New York. (1973)

[6] Abrams, R. H. Jr., Yadlowsky, E. J. and Lashinsky H.: periodic pulling and turbulence in a bounded plasma. Physical Review Letters, Vol. 22, Iss. 7-17 (1969)

[7] Berezin A. K. et al.: High-frequency oscillations excited by the interaction of an electron beam with a plasma. J. Nucl. Energy, Part C Plasma Phys. 7,593 (1965)

[8] Gordon, B.J. and Stover, H. L.: Injection locking of punched-through-injection transit-time oscillators. Proc. IEEE Vol.61 Iss. 12 (1973).

[10] Tomlinson, W. J. and Fork, R. L.: Properties of Gaseous Optical Masers in Weak Axial Magnetic Fields. Phys. Rev. 164, 466 (1967).

[11] Zhu, Z. and Piepmeier, E. H.: studies of mechanisms in glow discharge oscillations. Spectrochimica Acta Part B Vol.49 Iss. 12-14 (1994)

[12] Sitenko, A. G.: Nonlinear Wave Interaction and Fluctuations in Plasmas.

Phys. Scr. 1982, 67 (1982)

[13] Donahue, T. and. Dieke, G. H: Oscillatory Phenomena in Direct Current Glow Discharges. physical review 81,248 (1951)

[14] Petrovic, Z. L. and Phelps, A. V.: Osillations of Low Current Electrical Discharges Between Parallel Plane Electrodes I.D. Discharges. Physical Review E (4),2806 (1993)

[15] Nema, R. S. and Shrivastava, R. K.: Oscillatory Characteristics of a Low Pressure DC Discharge in Air. Journal of Physics D. Vol. 6 No.16 (1973)

[16] Jovanovic, A. P.: Electrical breakdown in air: new experiments and statistical and numerical models. Journal of Physics. Conference Series 565 - 012015 (2014)

[17] Georgi, P. T.: Fourier Series. Dover Publications Inc. (1976)

[18] Raizer, Yu. P.: Gas Discharge Physics. Berlin Springer (1991)

[19] Howatson, A. M.: An Introduction to Gas Discharges. Pergamon Press (1979)

[20] Wagenaars, E.: Plasma breakdown of low-pressure gas discharges. PhD Thesis. Technische University Eindhoven, Netherlands (2006) 